\title{
CORRESPONDENCE Cobrotoxin could be an effective therapeutic for COVID-19
}

\author{
Fang Lin ${ }^{1}$, Paul F. Reid ${ }^{2}$ and Zheng-hong Qin ${ }^{1}$ \\ Acta Pharmacologica Sinica (2020) 41:1258-1260; https://doi.org/10.1038/s41401-020-00501-7
}

Dear Editor,

A novel coronavirus, severe acute respiratory syndrome coronavirus 2 (SARS-CoV-2), which causes coronavirus disease 2019 (COVID-19), was declared a pandemic in 2020. It is generally agreed that the inflammatory cytokine storm, which causes systemic inflammation leading to multiple organ failures, is an important pathogenic mechanism in severe cases of COVID-19 [1]. Based on previous studies of cobra venom by the authors and other independent researchers, cobrotoxin, a short-chain aneurotoxin from Naja naja atra venom (NNAV), could be an alternative therapy for COVID-19.

Historically, cobra venom has been used as a traditional folk medicine for the treatment of multiple illnesses. Cobrotoxin comprises 62 amino acid residues with a molecular weight of about $7 \mathrm{kDa}$. Cobrotoxin can bind to several subtypes of the nicotinic acetylcholine receptors (nAChRs) and also a few other receptors and ion channels [2]. The wide distribution of nAChRs may be the main cause of the multiple pharmacodynamic actions of NNAV and cobrotoxin, including analgesic, antiviral, antiinflammatory, immunoregulatory, and antitumor activities.

\section{ANTI-INFLAMMATORY AND IMMUNOREGULATORY ACTIVITIES OF NNAV AND A-NEUROTOXINS}

It has been reported that NNAV and a-neurotoxins have antiinflammatory and immune regulatory actions. In numerous animal models and human clinical studies, cobra venom has demonstrated useful anti-inflammatory activity. Cobrotoxin can inhibit the inflammatory process in rat models of rheumatoid arthritis induced by adjuvant [3]. Cobratoxin, also an a-neurotoxin, has analgesic effects in rodent models of inflammatory pain induced by formalin and acetate [4]. Cobrotoxin also can inhibit the accumulation of lymphocytes and macrophages and the excretion of pro-inflammatory cytokines induced by lipopolysaccharides (LPS) (Wang SZ, unpublished).

Nuclear factor-KB (NF-KB) is a transcription factor that regulates the expression of genes involved in inflammatory responses and cell survival. Under nonstimulated conditions, NF-KB localizes to the cytoplasm through an interaction with inhibitory proteins of $K B$ family (IKBs). Under pro-inflammatory stimuli, IKBs are rapidly phosphorylated by IKB kinase (IKK) and degraded by the proteasome, which results in the release of free NF-KB dimers (p50 and Rel A) that translocate to the nucleus, to regulate the expression of target genes. Cobrotoxin binds to the kinase domain of IKK and inhibits its phosphorylation, preventing the release of free NF-KB from IKB. Cobrotoxin also binds with the critical cysteine residue (Cys-62) of $\mathrm{p} 50$ in the $\mathrm{N}$-terminal region of its DNA-binding domain to prevent NF-KB binding to DNA, thereby reducing the transcription of inflammatory genes [5]. We also found that cobrotoxin and cobra venom inhibit the nuclear location of NF-KB in several independent experiments $[3,6,7]$.

In addition to inflammation inhibitory effects, NNAV also has immune regulatory effects. NNAV can increase the activity of natural killer cells in normal mice, increase the secretion of interferon (IFN)- $\gamma$ and interleukin (IL)-4, promote CD4 Tlymphocyte differentiation into Th1 and Th2 subsets, and increase B-lymphocyte proliferation stimulated by LPS, as well as antibody production in response to sheep red blood cells [7]. NNAV markedly decreases T-lymphocyte proliferation stimulated by concanavalin A, suppresses CD4 and CD8 T-lymphocyte division, and inhibits IL-17 secretion and Th17 $\left(\mathrm{CD}^{+} \mathrm{CD} 4^{+} \mathrm{IL}-17^{+} \mathrm{T}\right)$ cell differentiation. Although NNAV decreases the numbers of both CD4 and CD8 T cells, the decrease in CD8 T cells is much more robust than that of CD4 T cells, resulting in restoration of the ratio of CD4/CD8. NNAV also prevents the decrease in serum immunoglobulin lgG and IgM concentrations in immunosuppressed mice [7].

\section{BROAD-SPECTRUM ANTIVIRAL ACTIVITIES OF COBRA VENOM AND A-NEUROTOXIN}

Previous studies have reported that a-neurotoxins inhibited replication and toxicity of several virus in both in vitro and in vivo. a-Neurotoxins have sequence homology to viral glycoproteins, including glycoprotein of rabies virus and gp120 of human immunodeficiency virus (HIV), which can specifically prevent the binding of the virus to nAChRs on cells and inhibit rabies virus [8] and HIV [9] infection. Cobra venom can also upregulate the gene transcription of type I IFNs. Viruses may have developed immune escape mechanisms based on selective inhibition of some IFN-a subtypes [10]. Therefore, the increase in type I IFN expression could be important for enhancing the immune system to prevent viral infection.

Cobra venom showed great efficacy in treating a patient with multidrug-resistant HIV. After subcutaneous injection of $0.1 \mathrm{~mL}$ cobra venom preparation daily (trade name Bioven) for 1 month, the viral load of the patient decreased from 1,580,000 copies $/ \mathrm{mL}$ to 3274 copies $/ \mathrm{mL}$ and the CD4 count increased from $52 / \mathrm{mm}^{3}$ to $324 / \mathrm{mm}^{3}$ [11]. The peptide derived from oxidative detoxification of a-neurotoxin could reduce herpes virus replication in infected brain tissues and increase survival time by $50 \%$ in mice [12]. In addition, cobrotoxin has a certain similarity to ACE2 according to sequence alignment results. ACE2 is the receptor through which the spike protein of SAR-COV-2 can bind to human cells [13]. The sequence similarity may suggest a possibility that NNAV or cobrotoxin could compete with SARS-COV-2 for binding to ACE2.

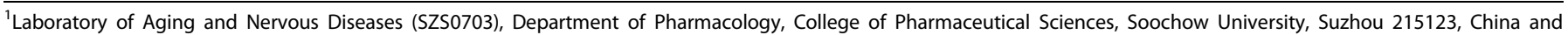
${ }^{2}$ Maitland Pharma, Orlando, Florida, USA

Correspondence: Zheng-hong Qin (qinzhenhong@suda.edu.cn)
}

Received: 23 June 2020 Accepted: 30 July 2020

Published online: 25 August 2020 
EFFECTS OF NNAV AND A-NEUROTOXIN ON INFLAMMATORY LUNG DISEASE

NNAV and a-neurotoxins have potent therapeutic effects in inflammation-induced lung diseases. Pulmonary fibrosis is a progressive and lethal lung disease characterized by the accumulation of extracellular matrix and the loss of pulmonary function. In the authors' early research on cobra venom, it was found that NNAV can improve lung gas exchange functionality and attenuate fibrotic lesions in the lung. In pulmonary fibrosis models induced by liposaccharide or bleomycin, NNAV showed the potential to decrease the levels of the inflammatory markers $\mathrm{IL}-1 \beta$ and tumor necrosis factor (TNF)- $\alpha$ in the serum. It was also found that NNAV inhibited the inflammatory process through inhibition of NF-KB in the LPS-induced model and the transforming growth factor- $\beta / S m a d$ pathway in the bleomycin-induced model [6]. In addition, cobrotoxin was shown to attenuate LPSinduced pulmonary edema, decrease the number of hematological $\mathrm{CD}^{+}{ }^{+} \mathrm{T}$ cells, inhibit immune cell accumulation in bronchoalveolar lavage fluid, and inhibit pro-inflammatory cytokine excretion in rat acute lung injury and acute respiratory distress syndrome [6]. Furthermore, it was also demonstrated that cobrotoxin can relieve chronic pulmonary fibrosis in rats (Wang SZ, unpublished).

\section{POTENTIAL USEFULNESS OF COBROTOXIN FOR THE MANAGEMENT OF COVID-19}

Patients with COVID-19 have persistently high levels of IL-6, TNFa, IL-1 $\beta, I L-8, I L 2 R$, and cytotoxic peptides such as perforin and granulysin [14]. The resulting inflammatory cytokine storm exacerbates lung damage in addition to other fatal complications. Therefore, in addition to inhibition of viral replication, antiinflammatory therapy is an important approach for combating COVID-19 morbidity. Lymphocytopenia is one of the most prominent markers of COVID-19 [15]. Lymphocytes are directly invaded by the virus or indirectly damaged by the subsequent cytokine storm. Corticosteroids are strong anti-inflammatory medications, but they also hamper the elimination of the virus by the immune system and increase the risk of secondary infections, mortality, and pulmonary fibrosis in survivors [16]. Some biological agents only target certain proinflammatory cytokines, leaving others unchecked, and thus have a limited impact on the cytokine storm. In addition, some anti-inflammatory medications inhibit the production of IFN-a, which may impede viral clearance. Therefore, drugs that have broad-spectrum anti-inflammatory and antiviral activities are valuable.

At this time, it is believed that cobrotoxin has the potential to treat patients with COVID-19 or to inhibit SARS-COV-2 infection. This conclusion is based on the following:

1. Anti-inflammatory activity: NNAV and a-neurotoxins have strong inhibitory effects on inflammation; thus, they could inhibit the cytokine storm caused by SARS-COV2 infection. In addition, the symptoms of patients with COVID-19 infection include deep vein thrombosis in hospitalized patients, which leads to serious adverse outcomes such as heart attack and stroke [17]. Inflammation and other factors contribute to a hypercoagulable state in COVID-19. NNAV and cobrotoxin can inhibit inflammation and possibly inhibit thrombosis caused by inflammation in both arteries and veins.

2. Immunoprotective activity: The numbers of white blood cells and lymphocytes are significantly reduced in the peripheral blood samples of patients infected with SARSCoV-2. The cellular immune responses triggered by COVID19 also develops through the overexpression of CD8 and hyperactivation of cytotoxic T lymphocytes [18]. NNAV and cobrotoxin inhibit the proliferation of CD8 T cells more than that of CD4 $\mathrm{T}$ cells and thus restore the CD4/CD8 ratio. NNAV also increases the concentration of serum IgG and $\operatorname{lgM}$ in mice with dexamethasone-induced immunosuppression, suggesting that NNAV or cobrotoxin could have the potential to restore the immune balance in patients with COVID-19.

3. Lung protection properties: Three stages of COVID-19 have been identified as viral infection, pulmonary inflammation, and fibrosis [19]. As pulmonary fibrosis causes irreversible fatal respiratory failure, antifibrotic therapies that prevent pulmonary fibrosis should be considered [20]. NNAV and cobrotoxin can inhibit lung inflammation, improve lung gas exchange function, and attenuate the development of fibrotic lesions in the lung.

4. Analgesic effect: Some patients with COVID-19 experience muscle pain and headache. NNAV and cobrotoxin are effective analgesics [21] that provide relief to patients with muscle pain and headache.

5. Antiviral activity: NNAV and a-neurotoxins have broadspectrum antiviral activities, although no direct evidence is available that NNAV or cobrotoxin could inhibit COVID-19.

6. Safety: Cobra venoms have a long history of clinical use as an analgesic and were first approved by the US Food and Drug Administration in the 1940s. Cobratide, an aneurotoxin from cobra venom, was approved as an analgesic in China and has been used safely since 1978. The $L_{50}$ of NNAV administered intragastrically in mice is $102 \mathrm{mg} / \mathrm{kg}$ and the $\mathrm{LD}_{50}$ of subcutaneously injected cobrotoxin in mice is $60 \sim 70 \mu \mathrm{g} / \mathrm{kg}$. Furthermore, subcutaneous injection of $2 \sim 6 \mu \mathrm{g} / \mathrm{kg}$ cobrotoxin has good anti-inflammatory effects in mice. Chronic toxicity data show that intragastric administration of $60 \sim 240 \mu \mathrm{g} / \mathrm{kg}$ NNAV for 12 months is safe in mice. In pilot trials for clinical pain treatment, it was suggested that $2 \sim 20 \mu \mathrm{g}$ cobrotoxin by subcutaneous injection and $500 \mu \mathrm{g}$ NNAV by mouth produced satisfactory effects.

\section{CONCLUSION}

NNAV and cobrotoxin are safe and may be effective in preventing and alleviating the symptoms caused by COVID-19.

\section{ACKNOWLEDGEMENTS}

This work was supported by the Priority Academic Program Development of Jiangsu Higher Education Institutions (PAPD, YX13200111) and the funding for Jiangsu Key Laboratory of Translational Research and Therapy for Neuro-Psycho-Diseases (BM2013003).

\section{ADDITIONAL INFORMATION}

Conflict of interest: All authors declare no conflict interest.

\section{REFERENCES}

1. Huang C, Wang Y, Li X, Ren L, Zhao J, Hu Y, et al. Clinical features of patients infected with 2019 novel coronavirus in Wuhan, China. Lancet. 2020;395:497-506.

2. Kuo KW, Chang LS, Chang CC. The structural loop II of cobrotoxin is the main binding region for $\mathrm{nAChR}$ and epitope in the region is conformation-dependent. J Biochem. 1995;117:438-42.

3. Zhu Q, Huang J, Wang SZ, Qin ZH, Lin F. Cobrotoxin extracted from Naja atra venom relieves arthritis symptoms through anti-inflammation and immunosuppression effects in rat arthritis model. J Ethnopharmacol. 2016;194:1087-95.

4. Shi GN, Liu YL, Lin HM, Yang SL, Feng YL, Reid PF, et al. Involvement of cholinergic system in suppression of formalin-induced inflammatory pain by cobratoxin. Acta Pharmacol Sin. 2011;32:1233-8. 
5. Park MH, Song HS, Kim KH, Son DJ, Lee SH, Yoon DY, et al. Cobrotoxin inhibits NFkappa $\mathrm{B}$ activation and target gene expression through reaction with NF-kappa B signal molecules. Biochemistry. 2005;44:8326-36.

6. Cui K, Kou JQ, Gu JH, Han R, Wang G, Zhen X, et al. Naja naja atra venom ameliorates pulmonary fibrosis by inhibiting inflammatory response and oxidative stress. BMC Complement Alter Med. 2014;14:461.

7. Kou JQ, Han R, Xu YL, Ding XL, Wang SZ, Chen CX, et al. Differential effects of Naja naja atra venom on immune activity. Evid Based Complement Altern Med. 2014;2014:287631.

8. Lentz TL, Fu Y, Lewis P. Rabies virus infection of IMR-32 human neuroblastoma cells and effect of neurochemical and other agents. Antivir Res. 1997;35:29-39.

9. Peptide derived from cobra venom inhibits HIV infection. AIDS Read. 2000;10:282.

10. Razzuoli E, Franzoni G, Carta T, Zinellu S, Amadori M, Modesto P, et al. Modulation of type I interferon system by African swine fever virus. Pathogens. 2020;9:361. https://doi.org/10.3390/pathogens9050361.

11. Alrajhi AA, Almohaizeie A. Snake venom preparation for drug-resistant human immunodeficiency virus. Ann Saudi Med. 2008;28:292-3.

12. Yourist JE, Haines HG, Miller KD. Inhibition of herpes simplex virus replication by cobra alpha-neurotoxoid. J Gen Virol. 1983;64:1475-81.

13. Lan J, Ge J, Yu J, Shan S, Zhou H, Fan S, et al. Structure of the SARS-CoV-2 spike receptor-binding domain bound to the ACE2 receptor. Nature. 2020;581:215-20.
14. Xu Z, Shi L, Wang Y, Zhang J, Huang L, Zhang C, et al. Pathological findings of COVID-19 associated with acute respiratory distress syndrome. Lancet Respir Med. 2020;8:420-2.

15. Chen G, Wu D, Guo W, Cao $Y$, Huang D, Wang $H$, et al. Clinical and immunological features of severe and moderate coronavirus disease 2019. J Clin Invest. 2020;130:2620-9.

16. Russell CD, Millar JE, Baillie JK. Clinical evidence does not support corticosteroid treatment for 2019-nCoV lung injury. Lancet. 2020;395:473-5.

17. Zhang L, Feng $X$, Zhang D, Jiang $C$, Mei H, Wang J, et al. Deep vein thrombosis in hospitalized patients with coronavirus disease 2019 (COVID-19) in Wuhan, China: prevalence, risk factors, and outcome. Circulation. 2020;142:114-28.

18. Ganji A, Farahani I, Khansarinejad B, Ghazavi A, Mosayebi G. Increased expression of CD8 marker on T-cells in COVID-19 patients. Blood Cells Mol Dis. 2020;83:102437.

19. Polak SB, Van Gool IC, Cohen D, von der Thusen JH, van Paassen J. A systematic review of pathological findings in COVID-19: a pathophysiological timeline and possible mechanisms of disease progression. Mod Pathol. 2020; 1-11. https://doi. org/10.1038/s41379-020-0603-3.

20. George PM, Wells AU, Jenkins RG. Pulmonary fibrosis and COVID-19: the potential role for antifibrotic therapy. Lancet Respir Med. 2020;8:807-15.

21. Chen ZX, Zhang HL, Gu ZL, Chen BW, Han R, Reid PF, et al. A long-form alphaneurotoxin from cobra venom produces potent opioid-independent analgesia. Acta Pharmacol Sin. 2006;27:402-8. 